\title{
Tinnitus- and Task-Related Differences in Resting-State Networks
}

\author{
Cris Lanting, Aron Woźniak, Pim van Dijk and Dave R. M. Langers
}

\begin{abstract}
We investigated tinnitus-related differences in functional networks in adults with tinnitus by means of a functional connectivity study. Previously it was found that various networks show differences in connectivity in patients with tinnitus compared to controls. How this relates to patients' ongoing tinnitus and whether the ecological sensory environment modulates connectivity remains unknown. Twenty healthy controls and twenty patients suffering from chronic tinnitus were enrolled in this study. Except for the presence of tinnitus in the patient group, all subjects were selected to have normal or near-normal hearing. fMRI data were obtained in two different functional states. In one set of runs, subjects freely viewed emotionally salient movie fragments ("fixed-state") while in the other they were not performing any task ("resting-state"). After data pre-processing, Principal Component Analysis was performed to obtain 25 components for all datasets. These were fed into an Independent Component Analysis (ICA), concatenating the data across both groups and both datasets, to obtain group-level networks of neural origin, each consisting of spatial maps with their respective time-courses. Subject-specific maps and their time-course were obtained by back-projection (Dual Regression). For each of the components a mixed-effects linear model was composed with factors group (tinnitus vs. controls), task (fixed-state vs. resting state) and their interaction. The neural components comprised the visual, sensorimotor, auditory, and limbic systems, the default mode, dorsal attention, executive-control, and frontoparietal networks, and the cerebellum. Most notably, the default mode network (DMN) was less extensive and shows significantly less connectivity in tinnitus patients than in controls. This group difference existed in both paradigms. At the same time, the DMN was stronger during resting-state than during fixed-state in the controls but not the patients. We attribute this pattern to the unremitting engaging effect of the tinnitus percept.
\end{abstract}

C. Lanting $(\varangle) \cdot$ P. van Dijk $\cdot$ D. R. M. Langers $\cdot$ A. Woźniak Department of Otorhinolaryngology / Head and Neck Surgery, University of Groningen, University Medical Center Groningen, Groningen, The Netherlands e-mail: c.p.lanting@umcg.nl

Graduate School of Medical Sciences (Research School of Behavioural and Cognitive Neurosciences), University of Groningen, Groningen, The Netherlands 
Keywords Tinnitus - Resting-state fMRI - Independent component analysis · Functional connectivity

\section{Introduction}

Tinnitus is a percept of sound that is not related to an acoustic source outside the body. For many forms of tinnitus, mechanisms in the central nervous system are believed to play a role in the pathology. Despite its high prevalence of 5-10\%, relatively little is known about the neural mechanisms and causes of tinnitus. Neuroimaging methods have been applied in the last decade to study potential mechanisms, and techniques such as electroencephalography (EEG), magnetoencephalography (MEG), positron emission tomography (PET), and functional magnetic resonance imaging (fMRI) have been extensively used to investigate neural correlates of tinnitus. Previously, neuroimaging studies on tinnitus relied mostly on task-related paradigms, such as presentation of sound stimuli that elicit sound-evoked responses, or manipulation of patients' tinnitus by somatic modulation (i.e., jaw protrusion or a change of gaze) to temporarily affect patients' tinnitus (Lanting et al. 2009). Alternatively, studies focused on anatomical differences in brain structure (Adjamian et al. 2014).

However, since a few years, new data-analysis techniques, such as independent component analysis (ICA), have emerged allowing for identification of coherent patterns of spatially independent, temporally correlated signal fluctuations in neuroimaging data during "resting-state", that is, without a predefined task. These patterns have since been termed "resting-state networks" (RSNs) (Greicius et al. 2004) and represent functionally relevant and connected networks or systems supporting core perceptual and cognitive processes. Resting-state networks are generally reported to show reliable and consistent patterns of functional connectivity (Zhang et al. 2008).

Moreover, advances in data-analysis currently allow for between-group analyses where individual networks can be compared across tasks and groups. Recently, resting-state fMRI has been used to investigate functional connectivity differences in individuals with tinnitus (Burton et al. 2012; Davies et al. 2014; Kim et al. 2012; Maudoux et al. 2012a, b). Results indicate differences in connectivity between healthy and affected subject groups. One result showed reduced connectivity between the left and right auditory cortex, indicative of disturbed excitation and inhibition across hemispheres (Kim et al. 2012). A different study found that in individuals with tinnitus connectivity was increased in numerous brain regions, including the cerebellum, parahippocampal gyrus, and sensorimotor areas. Decreased connectivity was found in the right primary auditory cortex, left prefrontal, left fusiform gyrus, and bilateral occipital regions (Maudoux et al. 2012a, b). Very recently, altered interhemispheric functional connectivity was shown and linked with specific tinnitus characteristics in chronic tinnitus patients (Chen et al. 2015a). In addition, enhanced functional connectivity between auditory cortex and the homologue 
frontal gyrus was observed, indicative of enhanced connectivity of auditory networks with attention networks (Chen et al. 2015b). All these results combined are thought to represent the influence tinnitus has on the networks encompassing memory, attention and emotion. Whether this is causal and which direction the causality goes remains unknown.

In this chapter we describe tinnitus-related changes in functional networks in adults with tinnitus compared to adults without tinnitus. We aim to study whether group-differences can be observed in functional connectivity at the group level and whether performance of an engaging task alters these connectivity patterns.

\section{Methods}

\subsection{Participants}

Twenty healthy controls and twenty patients suffering from chronic tinnitus were enrolled in this fMRI study after providing written informed consent, in accordance with the medical ethical committee at the University Medical Center Groningen, the Netherlands. Subjects were recruited from the hospital's tinnitus outpatient clinic as well as from advertisements in local media. None of them had a history of neurological or psychiatric disorders. Subjects' characteristics are listed in Table 1 (Langers et al. 2012). Except for the presence of tinnitus in the patient group, all subjects were selected to have normal or near-normal hearing up to $8 \mathrm{kHz}$. Thresholds were determined in a frequency range of $0.25-16 \mathrm{kHz}$ by means of pure-tone audiometry. Subjects also performed the adaptive categorical loudness scaling (ACALOS) procedure (Brand and Hohmann 2002). To characterize the participants' self-reported complaints, all subjects filled out the 14-item Hyperacusis Questionnaire, relating to the attentional, social, and emotional aspects of auditory hypersensitivity (Khalfa et al. 2002).

The tinnitus patients also filled out Dutch translations of questionnaires related to their tinnitus, including the Tinnitus Handicap Inventory (THI) measuring tinnitus severity in daily life (Newman et al. 1996), the Tinnitus Reaction Questionnaire (TRQ) that assesses the psychological distress associated with tinnitus (Wilson et al. 1991), and the Tinnitus Coping Style Questionnaire (TCSQ) that quantifies effective as well as maladaptive coping strategies (Budd and Pugh 1996). Patients were asked about the laterality of their tinnitus and the character of the tinnitus sound. Using a modified tinnitus spectrum test, patients were asked to indicate the "likeness" of their tinnitus to narrow-band noises of varying center frequencies. 
Table 1 Several group characteristics. Numbers indicate mean \pm standard deviation (range). All questionnaire scales were expressed to range from 0 to 100 for ease of interpretation. The significance of group differences was based on Fisher's exact test (for gender and handedness) or Student's t-test (for all other comparisons), and classified as: $* * * p<0.001 ; * * p<0.01 ;{ }^{*} p<0.05$; ${ }^{\#} \mathrm{p} \geq 0.05$. Numbers do not add up to equal the group size because for some patients not all tinnitus characteristics were obtained

\begin{tabular}{|c|c|c|c|c|}
\hline Group & & Healthy controls & Tinnitus patients & $p$ \\
\hline & & $(n=20)$ & $(n=20)$ & \\
\hline \multicolumn{5}{|c|}{ Demographics } \\
\hline & Gender & 16 female, 4 male & 12 female, 8 male & \# \\
\hline & Handedness & 17 right, 3 left & 19 right, 1 left & \# \\
\hline & Age (years) & $33 \pm 13(21-60)$ & $46 \pm 11(26-60)$ & $* *$ \\
\hline \multicolumn{5}{|c|}{ Audiometry } \\
\hline & $\begin{array}{l}\text { Average threshold (dB } \\
\text { HL) }\end{array}$ & $5 \pm 5(-1-18)$ & $8 \pm 5(0-23)$ & $\#$ \\
\hline & Loudness range $(\mathrm{dB})$ & $98 \pm 8(84-113)$ & $84 \pm 14(56-105)$ & $* * *$ \\
\hline \multicolumn{5}{|c|}{ Self-reported symptoms } \\
\hline & Hyperacusis (\%) & $25 \pm 14(8-51)$ & $59 \pm 16(29-82)$ & $* * *$ \\
\hline & Depression (\%) & $8 \pm 9(0-31)$ & $27 \pm 27(0-100)$ & $* *$ \\
\hline & Dysthymia (\%) & $18 \pm 14(0-44)$ & $38 \pm 24(0-75)$ & $* *$ \\
\hline \multicolumn{5}{|c|}{ Tinnitus effects } \\
\hline & Tinnitus Handicap (\%) & - & $43 \pm 22(6-88)$ & - \\
\hline & Tinnitus Reaction (\%) & - & $38 \pm 21(0-88)$ & - \\
\hline & Effective Coping (\%) & - & $54 \pm 14(17-68)$ & - \\
\hline & Maladaptive Coping (\%) & - & $29 \pm 15(3-60)$ & - \\
\hline \multicolumn{5}{|c|}{ Tinnitus percept } \\
\hline & Lateralization & - & \begin{tabular}{|l|}
8 central, 4 right, \\
2 left
\end{tabular} & - \\
\hline & Type & - & $\begin{array}{l}16 \text { steady, } 3 \\
\text { pulsatile }\end{array}$ & - \\
\hline & Bandwidth & - & $\begin{array}{l}10 \text { tone, } 7 \text { hiss, } \\
2 \text { ring }\end{array}$ & - \\
\hline & Frequency & - & $\begin{array}{l}16 \text { high, } 2 \text { other, } \\
1 \text { low }\end{array}$ & - \\
\hline
\end{tabular}

\subsection{Imaging Paradigm}

The equipment used for this study was a 3.0-T MR scanner (Philips Intera, Best, the Netherlands), supplied with an 8-channel phased-array (SENSE) transmit/receive head coil. The functional imaging sessions performed on subjects in head-first-supine position in the bore of the system included two runs for the resting-state and two runs for the fixed-state designs. Each resting-state run consisted of a dynamic series of 49 identical high-resolution T2*-sensitive gradient-echo echo-planar imaging (EPI) volume acquisitions (TR $15.0 \mathrm{~s}$; TA $2.0 \mathrm{~s}$; TE $22 \mathrm{~ms}$; FA 90; matrix $64 \times 64 \times 48$; resolution $3 \times 3 \times 3 \mathrm{~mm}^{3}$; interleaved slice order, no slice gap), whereas fixed-state runs consisted of the same acquisition parameters except the TR that for 
the fixed-state runs was equal to the TA of $2.0 \mathrm{~s}$ (continuous scanning) and an ascending slice ordering. The acquisition volume was oriented parallel to the Sylvian fissure, and centered approximately on the superior temporal sulci. In total were 361 volumes acquired.

A sparse, clustered-volume sequence was employed in the (unstimulated) resting-state runs to avoid interference from acoustic scanner noise, while continuous acquisitions were employed in the (stimulated) fixed-state runs to provide higher temporal resolution and power. The scanner coolant pump was turned off during to further reduce noise-levels. In order to control subjects' attentional state, they were asked to focus on the fixation cross throughout the imaging time for resting-state runs, or watch four "emotional" 3-min movie fragments (video and audio) for fixedstate runs, while being scanned simultaneously.

To ensure that the ambient noise did not eliminate the patients' tinnitus, they were asked to rate their tinnitus several times (before, between and after imaging runs) on a $0-10$ scale, where 0 indicated no perception of tinnitus at all and 10 indicated the strongest tinnitus imaginable. Ratings did not decrease significantly throughout the session (conversely, some patient's indicated that it systematically increased with stress and fatigue) and never reached zero ( 2 was the lowest rating).

\subsection{Stimuli}

MR-compatible electrodynamic headphones (MR Confon GmbH, Magdeburg, Germany; (Baumgart et al. 1998)) were used during the fixed-state runs to deliver sound stimuli from a standard PC with soundcard to participants. Subjects were asked to wear foam earplugs underneath the headset to further diminish the acoustic noise created by the scanner. Visual stimuli were presented by means of a built-in MR-compatible LCD display.

\subsection{Preprocessing}

Nipype (Gorgolewski et al. 2011) was used to preprocess the fMRI data. Preprocessing consisted of realignment (FSL), artifact rejection (RapidArt) and spatiotemporal filtering to reduce physiological noise (Behzadi et al. 2007). Further preprocessing steps included band-pass filtering $(0.1-1.0 \mathrm{~Hz})$, registration to an individual highresolution T1-weighted anatomical image and normalization into MNI stereotaxic space using ANTs (Klein et al. 2009). The data were resampled to a 3-mm isotropic resolution and moderately smoothed using an isotropic 5-mm FWHM Gaussian kernel to improve signal to noise ratio. 


\subsection{Group Analysis}

Next, Principal Component Analysis (PCA) was performed to obtain 25 components for each dataset and each subject separately, explaining on average $79.8 \%$ of the total variance. These components were subsequently fed into a group Probabilistic Independent Component Analysis as implemented in FSL's MELODIC (v. 3.14) using multi-session temporal concatenation. The obtained networks consisted of spatial maps with their respective time-courses. Subject-specific maps and their time-courses were obtained by back-projection using FSL's Dual Regression. This method uses two stages of regression where in a first stage the individual timecourses are obtained that best fit the group maps. In a second stage, the raw data is regressed against these time-courses to obtain subject-specific component-maps corresponding to the overall group-map.

Importantly, the outputs of stage one of the dual regression - the individual time-courses - were variance normalized before the second stage of the dual regression, allowing for assessment of the shape and amplitude of the component maps. Manual inspection of the time-courses and maps as well as the (spatial) correlation of the maps with previously reported resting-state networks (Smith et al. 2009) were used to distinguish meaningful components from components containing non-neural signals.

For each of the components, a mixed-effects linear model was composed in AFNIs 3dLME (Chen et al. 2013) with factors group (tinnitus vs. controls) and task (fixed-state vs. resting state) and their interaction. The subjects' component maps were fed into this analysis and the significance of the factors was assessed for each component. Further, we assessed the power spectral density (PSD) of the corresponding time-courses.

\section{Results}

A total of ten networks were identified as neural in origin (Fig. 1). Based on a spatial correlation coefficient with previous networks (Smith et al. 2009) we identified clear sensorimotor networks: two visual networks [Vis1, Vis2], one auditory network [Aud1], a somatosensory/motor network [Sens.Motor] and the cerebellum [Cereb]. In addition, typical resting-state networks were found, such as the limbic system [Limbic], the dorsal attention network [DAN1, DAN2], the default mode network [DMN] and a frontoparietal network [Front.Par].

From these group-level networks, we obtained subject-specific component-maps by applying a dual-regression technique (Filippini et al. 2009). This method uses two stages of regression, similar to back-projection of individual data to group maps used in other studies. By applying dual-regression we allow for variation between subjects, while maintaining the correspondence of components across subjects. 

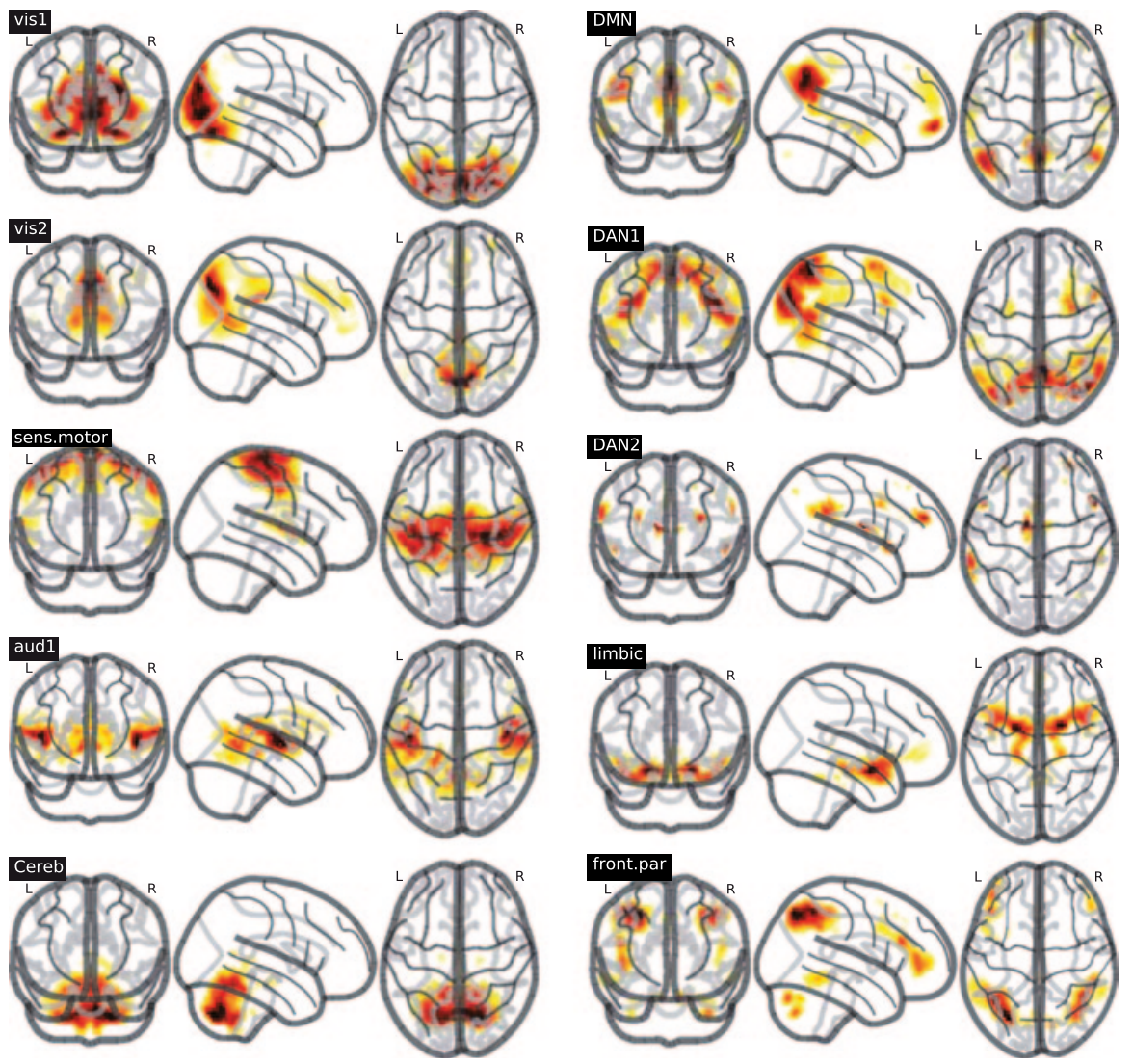

Fig. 1 Ten components obtained from the group analysis using multi-session temporal concatenation of all individual data. Each of the components is thresholded at $z>3$. The maps in the left column are classical task-related sensory networks while those in the right column show typical resting-state networks

The subject-specific maps were subsequently fed into a linear mixed model (AFNI's 3dLME) with factors group and task. Importantly, the outputs of stage one of the dual regression - the individual time-courses - were variance normalized before the second stage of the dual regression. In this manner the linear-mixed model now tests for both differences between groups in the shape of the network as well as in the amplitude of the network. The results from these analyses indicated that several networks behaved differently in controls than in subjects with tinnitus. Most notably it identified the default mode network (DMN), roughly encompassed by two distinct but similar networks in the data

Figure 2 shows the two networks that partly overlap. Panel A indicates the DMN that also correlates well with previous demarcations of the DMN (spatial correlation $=0.8$ ) (Smith et al. 2009). In addition, panel E shows a component consisting of a visual area and the posterior and anterior cingulate gyrus. In both the DMN 


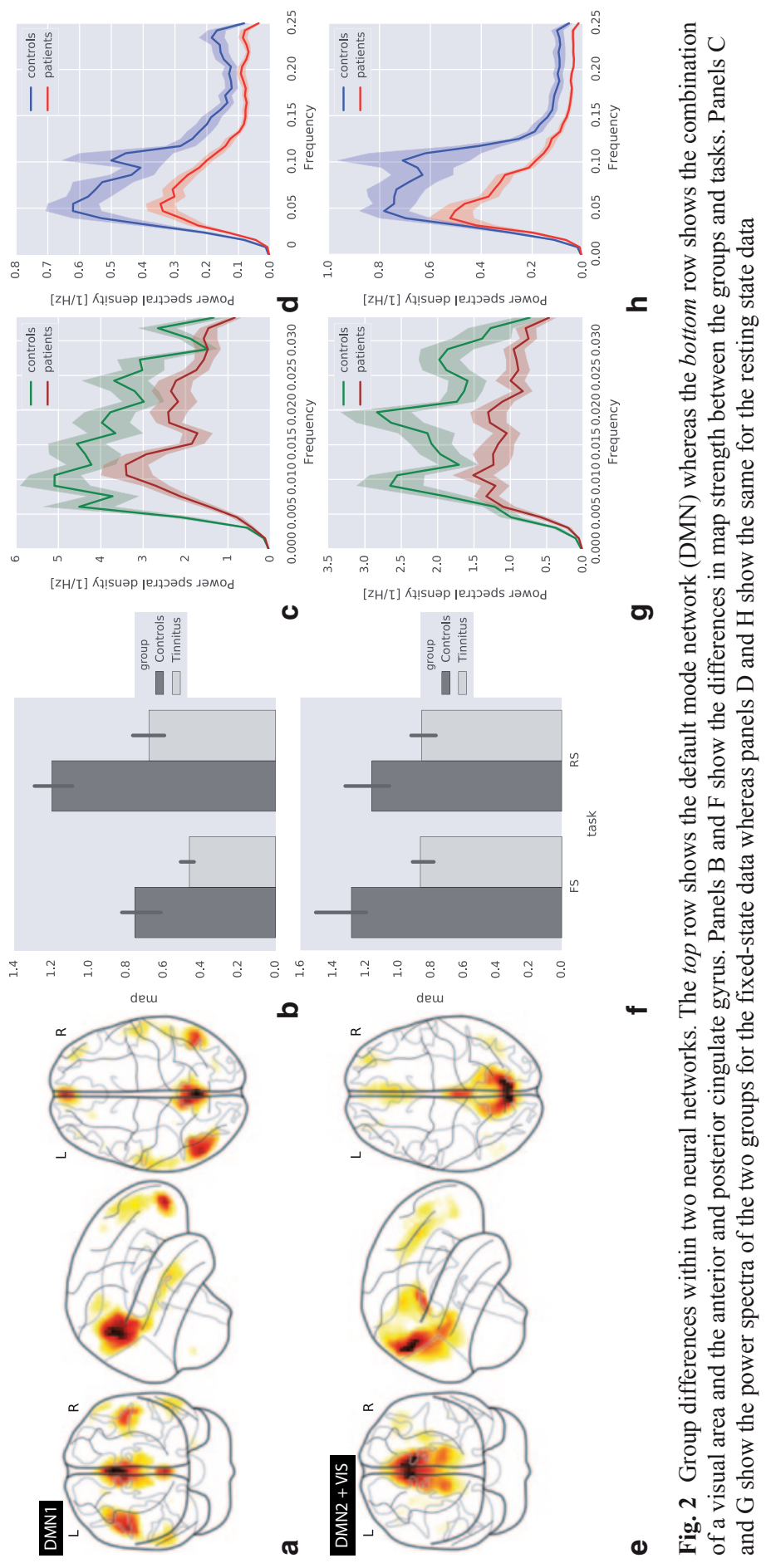




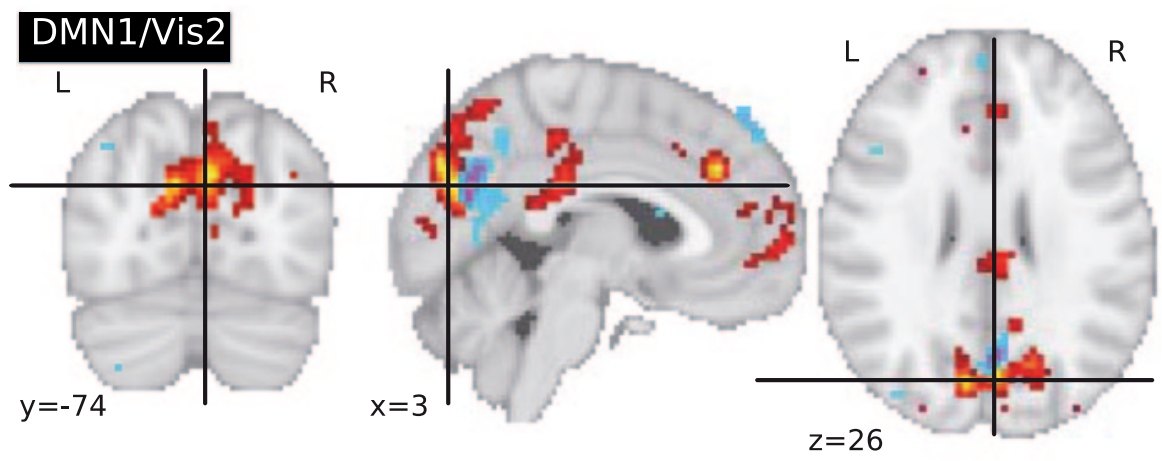

Fig. 3 Group differences within two neural networks. The red-yellow colours indicate the significant differences in the network representing a visual area and the cingulate gyrus (see Fig. 2e), and the blue-purple colours indicate the differences in the default-mode network (Fig. 2a). These areas are remarkably adjacent, corresponding to the left and right (pre)cuneus. This area shows significantly reduced functional connectivity in patients compared to controls in both networks

and the latter component, there are adjacent voxels that show a clear and significant group difference $(p<0.001$ for each of the maps, corresponding to a F-value of 12.71). When looking at the map strength, a proxy for functional connectivity, patients consistently show decreased functional connectivity (panels B and F). Moreover, whereas controls show a clear task-effect, as would be expected for the DMN, this task-effect is much smaller in patients (panel B). For the network with the visual cortex and cingulate gyrus (panel E), this task-effect is less clear (panel F) and shows in controls a decrease during resting state compared to watching a movie, presumably due to the lack of visual stimulation throughout the acquisition of resting-state data. The pattern of reduced connectivity is also clearly visible as a decreased power in the time-courses of the same voxels. This is shown for the both the fixed-state data (panels $\mathrm{C}$ and $\mathrm{G}$ ) and the resting-state data (panels D and F). In all cases, the power is significantly lower in the patient groups.

Figure 3 shows the areas where the subject groups differ significantly are almost if not completely adjacent, indicating the potential relevance of the two spatial networks in tinnitus.

\section{Discussion}

This study shows that by using resting-state fMRI in combination with independent component analysis we are able to detect differences in neural networks between substantially-sized subject groups. Resting-state fMRI is a valuable addition to traditional task-related fMRI, where a difference between conditions is obtained. This might not necessarily be the best paradigm to study tinnitus. The consequence of a task-related design is that, by using a subtraction approach, the tinnitus-related activity, present in both conditions, will not be present in the subtraction image unless the task affects the perception of tinnitus (Lanting et al. 2009). 
Our data analysis is different from other studies that have used resting-state fMRI to study tinnitus. Often the decomposition of the data into independent component is done by performing two (or more) separate ICA analyses on two (or more) groups (see e.g. Schmidt et al. 2013). This leads to a difficult interpretation since it is then unclear whether group differences represent (true) differences between subject groups, or whether the data-decomposition was slightly different (biases) for the two groups.

In contrast, we adopted a strategy where the data is pooled over all participants and data-modalities (resting- and fixed-state data). In this way, the data-decomposition is not determined in a potentially different way for both groups, leaving out the necessity of post-ICA matching of corresponding component maps. This allows for a better group-estimate of the components. In addition, by using a dual regression technique we can obtain subject-specific maps and perform group-wise analyses on these maps (Filippini et al. 2009).

This study shows that by using resting-state fMRI in combination with independent component analysis we are able to detect differences in neural networks between substantially-sized subject groups. Resting-state fMRI is a valuable addition to traditional task-related fMRI, where a difference between conditions is obtained. This might not necessarily be the best paradigm to study tinnitus. The consequence of a task-related design is that, by using a subtraction approach, the tinnitus-related activity, present in both conditions, will not be present in the subtraction image unless the task affects the perception of tinnitus (Lanting et al. 2009)

Our data analysis is different from other studies that have used resting-state fMRI to study tinnitus. Often the decomposition of the data into independent component is done by performing two (or more) separate ICA analyses on two (or more) groups (see e.g. (Schmidt et al. 2013)). This lead to a difficult interpretation since it is then unclear whether group differences represent (true) differences between subject groups, or whether the data-decomposition was slightly different (biases) for the two groups.

In contrast, we adopted a strategy where the data is pooled over all participants and data-modalities (resting- and fixed-state data). In this way, the data-decomposition is not determined in a potentially different way for both groups, leaving out the necessity of post-ICA matching of corresponding component maps. This allows for a better group-estimate of the components. In addition, by using a dual regression technique we can obtain subject-specific maps and perform group-wise analyses on these maps (Filippini et al. 2009). Using this methodological approach we found a significant group effects for the DMN.

Our interpretation is that the activity of the DMN in patients with tinnitus, whether as the source or the consequence of tinnitus, is reduced in comparison to the activity in controls. The DMN is relatively better connected internally (i.e. larger spontaneous fluctuations, indicative of stronger connectivity) when the mind is at rest and allowed to wander or daydream. This network is believed to be key in how a person introspectively understands themselves and others, and forms beliefs, intentions, and desires through autobiographical memory. Recently it was shown that a dysfunctional DMN is related to autism (Washington et al. 2014). Perhaps the 
DMN plays a similar role in tinnitus, where through the on-going tinnitus patients can't get into a state of mind-wandering and therefore show a reduced activity in the DMN compared to controls.

This DMN has been previously implicated in other diseases such as Alzheimer's disease. Lower functional connectivity scores in the DMN were found in an Alzheimer's disease group when compared to normal groups (Binnewijzend et al. 2012). In contrast, results of resting-state data-analysis in schizophrenia have been variable (Greicius 2008). Schizophrenia is regarded as a large cluster of profound neuropsychiatric symptoms. A subtype of patients (also) experience phantom perception of sounds. One interpretation is that, since the DMN is different between groups in previous work and ours, the DMN somehow plays a role in 'hearing' internally generated sound (whether it is meaningful like in patients with schizophrenia or meaningless like tinnitus).

Our study is novel in the sense that it specifically assesses the influence of performing a task on the scale of brain networks as a whole. Specifically in patients with tinnitus, the DMN does not clearly show a task-effect, which in contrast is clearly present in controls. This observation enhances the hypothesis that subjects with tinnitus cannot get the 'rest' that is necessary for a healthy life. In conclusion, we attribute the pattern of reduced connectivity in the DMN to the unremitting engaging effect of the tinnitus percept.

Acknowledgments DRML was funded by VENI research grant 016.096.011 from the Netherlands organization for scientific research (NWO) and the Netherlands organization for health research and development (ZonMw). Further financial support was provided by the Heinsius Houbolt Foundation.

Open Access This chapter is distributed under the terms of the Creative Commons AttributionNoncommercial 2.5 License (http://creativecommons.org/licenses/by-nc/2.5/) which permits any noncommercial use, distribution, and reproduction in any medium, provided the original author(s) and source are credited.

The images or other third party material in this chapter are included in the work's Creative Commons license, unless indicated otherwise in the credit line; if such material is not included in the work's Creative Commons license and the respective action is not permitted by statutory regulation, users will need to obtain permission from the license holder to duplicate, adapt or reproduce the material.

\section{References}

Adjamian P, Hall DA, Palmer AR, Allan TW, Langers DRM (2014). Neuroanatomical abnormalities in chronic tinnitus in the human brain. Neurosci Biobehav Rev 45:119-133

Baumgart F, Kaulisch T, Tempelmann C, Gaschler-Markefski B, Tegeler C, Schindler F, Scheich H (1998) Electrodynamic headphones and woofers for application in magnetic resonance imaging scanners. Med Phys 25(10):2068-2070

Behzadi Y, Restom K, Liau J, Liu TT (2007) A component based noise correction method (CompCor) for BOLD and perfusion based fMRI. Neuroimage 37(1):90-101. http://doi.org/10.1016/j. neuroimage.2007.04.042

Binnewijzend MAA, Schoonheim MM, Sanz-Arigita E, Wink AM, van der Flier WM, Tolboom N, Barkhof F (2012) Resting-state fMRI changes in Alzheimer's disease and mild cogni- 
tive impairment. Neurobiol Aging 33(9):2018-2028. http://doi.org/10.1016/j.neurobiolaging.2011.07.003

Brand T, Hohmann V (2002) An adaptive procedure for categorical loudness scaling. J Acoust Soc Am 112(4):1597-1604. http://doi.org/10.1121/1.1502902

Budd RJ, Pugh R (1996) Tinnitus coping style and its relationship to tinnitus severity and emotional distress. J Psychosom Res 41(4):327-335. http://doi.org/10.1016/S0022-3999(96)00171-7

Burton H, Wineland A, Bhattacharya M, Nicklaus J, Garcia KS, Piccirillo JF (2012) Altered networks in bothersome tinnitus: a functional connectivity study. BMC Neurosci 13(1):3. http:// doi.org/10.1186/1471-2202-13-3

Chen G, Saad ZS, Britton JC, Pine DS, Cox RW (2013) Linear mixed-effects modeling approach to fMRI group analysis. Neuroimage 73:176-190. http://doi.org/10.1016/j.neuroimage.2013.01.047

Chen Y, Zhang J, Li X, Xia W, Feng X, Qian C, Teng G (2015a) Altered intra- and interregional synchronization in resting-state cerebral networks associated with chronic tinnitus. Neural Plast 2015:1-11. http://doi.org/10.1155/2015/475382

Chen Y, Zhang J, Li X, Xia W, Feng X, Qian C, Teng G (2015b) Altered intra- and interregional synchronization in resting-state cerebral networks associated with chronic tinnitus. Neural Plast 2015:1-11. http://doi.org/10.1155/2015/475382

Davies J, Gander PE, Andrews M, Hall Da (2014) Auditory network connectivity in tinnitus patients: a resting-state fMRI study. Int J Audiol 53(3):192-198. http://doi.org/10.3109/149920 27.2013.846482

Filippini N, MacIntosh BJ, Hough MG, Goodwin GM, Frisoni GB, Smith SM, Mackay CE (2009) Distinct patterns of brain activity in young carriers of the APOE-epsilon4 allele. Proc Natl Acad Sci USA 106(17):7209-7214. http://doi.org/10.1073/pnas.0811879106

Gorgolewski K, Burns CD, Madison C, Clark D, Halchenko YO, Waskom ML, Ghosh SS (2011). Nipype: a flexible, lightweight and extensible neuroimaging data processing framework in python. Front Neuroinform 5(August):13. http://doi.org/10.3389/fninf.2011.00013

Greicius M (2008) Resting-state functional connectivity in neuropsychiatric disorders. Curr Opin Neurol 21(4):424-430. http://doi.org/10.1097/WCO.0b013e328306f2c5

Greicius MD, Srivastava G, Reiss AL, Menon V (2004) Default-mode network activity distinguishes Alzheimer's disease from healthy aging: evidence from functional MRI. Proc Natl Acad Sci USA 101(13):4637-4642. http://doi.org/10.1073/pnas.0308627101

Khalfa S, Dubal S, Veuillet E, Perez-Diaz F, Jouvent R, Collet L (2002) Psychometric normalization of a hyperacusis questionnaire. ORL 64:436-442. http://doi.org/10.1159/000067570

Kim J, Kim Y, Lee S, Seo J-H, Song H-J, Cho JH, Chang Y (2012). Alteration of functional connectivity in tinnitus brain revealed by resting-state fMRI?: a pilot study. Int J Audiol. http://doi. org/10.3109/14992027.2011.652677

Klein A, Andersson J, Ardekani B, Ashburner J, Avants B, Chiang M-C, Parsey RV (2009) Evaluation of 14 nonlinear deformation algorithms applied to human brain MRI registration. Neuroimage 46(3):786-802. http://doi.org/10.1016/j.neuroimage.2008.12.037

Langers DRM, de Kleine E, van Dijk P (2012). Tinnitus does not require macroscopic tonotopic map reorganization. Front Syst Neurosci 6:2. http://doi.org/10.3389/fnsys.2012.00002

Lanting CP, de Kleine E, van Dijk P (2009) Neural activity underlying tinnitus generation: results from PET and fMRI. Hear Res 255(1-2):1-13. http://doi.org/10.1016/j.heares.2009.06.009

Maudoux A, Lefebvre P, Cabay J-E, Demertzi A, Vanhaudenhuyse A, Laureys S, Soddu A (2012a) Auditory resting-state network connectivity in tinnitus: a functional MRI study. PloS One 7(5):e36222. http://doi.org/10.1371/journal.pone.0036222

Maudoux, A, Lefebvre P, Cabay J-E, Demertzi A, Vanhaudenhuyse A, Laureys S, Soddu A (2012b) Connectivity graph analysis of the auditory resting state network in tinnitus. Brain Res 1485:10-21. http://doi.org/10.1016/j.brainres.2012.05.006

Newman CW, Jacobson GP, Spitzer JB (1996). Development of the tinnitus handicap inventory. Arch Otolaryngol Head Neck Surg 122:143-148. http://doi.org/10.1001/archot ol.1996.01890140029007 
Schmidt SA, Akrofi K, Carpenter-Thompson JR, Husain FT (2013) Default mode, dorsal attention and auditory resting state networks exhibit differential functional connectivity in tinnitus and hearing loss. PloS One 8(10):e76488. http://doi.org/10.1371/journal.pone.0076488

Smith SM, Fox PT, Miller KL, Glahn DC, Fox PM, Mackay CE, Beckmann CF (2009) Correspondence of the brain's functional architecture during activation and rest. Proc Natl Acad Sci USA 106(31):13040-13045. http://doi.org/10.1073/pnas.0905267106

Washington SD, Gordon EM, Brar J, Warburton S, Sawyer AT, Wolfe A, Vanmeter JW (2014) Dysmaturation of the default mode network in autism. Hum Brain Mapp 35(4):1284-1296. http://doi.org/10.1002/hbm.22252

Wilson PH, Henry J, Bowen M, Haralambous G (1991) Tinnitus reaction questionnaire: psychometric properties of a measure of distress associated with tinnitus. J Speech Hear Res 34(1):197-201

Zhang D, Snyder AZ, Fox MD, Sansbury MW, Shimony JS, Raichle ME (2008) Intrinsic functional relations between human cerebral cortex and thalamus. J Neurophysiol 100(4):1740-1748. http://doi.org/10.1152/jn.90463.2008 